\title{
Optimal Threshold Selection for Wavelet Transform based on Visual Quality
}

\author{
Baby Vijilin \\ LBS Institute of Technology for Women, \\ Trivandrum, India
}

\author{
V.K.Govindan \\ Department of Computer Science and Engineering \\ National Institute of Technology Calicut, India
}

\begin{abstract}
Wavelet transform technique has been used for image compression targeting high visual quality reconstructed images even with high compression ratio. A visual quality measure such as Picture Quality Scale (PQS), which correlates well with the subjective Mean Opinion Score (MOS) may be employed on the compressed image for the quantizer to select the optimum dynamic threshold. The use of optimum threshold permits the removal of redundant information, thus leading to better compression performance with acceptable picture quality. The Results obtained with the proposed approach of threshold selection is compared with the existing technique and the performance and it is found to be better in all of the cases of images or wavelets.
\end{abstract}

\section{General Terms}

Image Compression, Wavelet Transform, Denoising, Threshold Selection

\section{Keywords}

Image Compression, Optimum Threshold, Visual quality, Wavelet Transform

\section{INTRODUCTION}

In recent years many approaches to image and signal denoising have been proposed [1]-[8]. Wavelet-based image denoising is an important technique in the area of image noise reduction [9]. Image compression researchers have shown active interest in adaptive wavelet image compression since early 1990's. One of the important works is that by M. L. Hilton and R. T. Ogden [10]. They proposed a data adaptive scheme for wavelet shrinkage-based noise removal. The method involves a statistical test of hypotheses that takes into account the magnitudes of wavelet coefficients and relative positions. The amount of smoothing performed during noise removal is controlled by the user-supplied confidence level of the tests. Paper [2] presented a denoising method for ultrasound medical images by linear filtering by Gaussian filters of 2D wavelet coefficients.

Another work is that by J. S. Weszka et al. [11] in which Laplacian operation is applied to the picture to determine points that lie on or near the edges of objects. Threshold selection becomes easier when the frequency distribution of gray levels of these points is used.

Wavelets are functions generated by a linear combination of the shifted and scaled version of a fixed function, called the mother wavelet, denoted by $\Psi$. Any function can be represented by superimposing translated and dilated versions of $\Psi$, denoted by $\Psi \mathrm{j}, \mathrm{i}$, where $\mathrm{i}$ and $\mathrm{j}$ are translation and dilation parameters. The $\Psi \mathrm{j}$, i can be computed from the mother wavelet as

$\Psi_{\mathrm{j}, \mathrm{i}}(\mathrm{x})=2^{\mathrm{j} / 2} \Psi\left(2^{\mathrm{j}} \mathrm{x}-\mathrm{i}\right)$
The wavelet transform is implemented by quadrature mirror filters, G, a low-pass filter, and $\mathrm{H}$, a high-pass filter. The Wavelet transform represents any function $f$ as superpositions of wavelets [12]. Detailed analysis of the relationship between wavelets and QMF is given in [13].

The Process of decomposing a function into wavelet coefficients is called wavelet transform. In discrete wavelet transform if the parameters $\mathrm{i}$ and $\mathrm{j}$ take discrete essentially leading to a finite number of coefficients. For images, the hierarchical wavelet decomposition suggested in [13] is used. The $\mathrm{G}$ and $\mathrm{H}$ filters are applied to the image in both the horizontal and vertical directions for decomposition. Thus, decomposition provides four sub bands corresponding to different resolution levels and orientation (Figure 1). (a)one-level

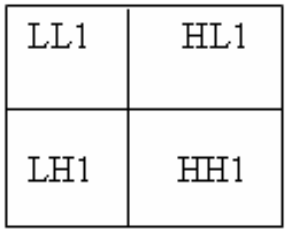

(b)two-level

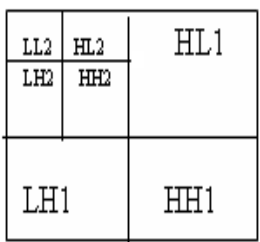

Fig:1 Image decomposition

The sub-bands labeled LH1, HL1, and HH1 are the selective high-pass sub-bands representing the finest scale wavelet coefficients (image details), whereas the sub-bands LL1 is the low pass sub-band corresponding to coarse level coefficients (approximation image).

To obtain the next coarse level of wavelet coefficients (two level wavelet decomposition) the sub-band LL1 is further decomposed leading to further sub-bands as in Figure 1(b). Similarly, to obtain further levels of decomposition, the process is repeated on the LL2 band and then on LL3 band etc.. Figure 2 shows the image of Cameraman decomposed in to 7 sub-bands.

When a signal is decomposed using the wavelet transform, it is left with a set of wavelet coefficients that correspond to the high frequency sub-bands. These high frequency sub-bands consist of the details in the data set. If these details are small enough, they may be omitted without substantially affecting the main features of the data set. Additionally, these small details are often those associated with noise; therefore, by setting these coefficients to zero, it is essentially eliminating the noise. This is the basic concept behind the use of thresholding for compressing the images. In this approach, all frequency sub-band coefficients that are less than a particular threshold are set to zero and the remaining coefficients are used in an inverse wavelet transformation to reconstruct the data set. 


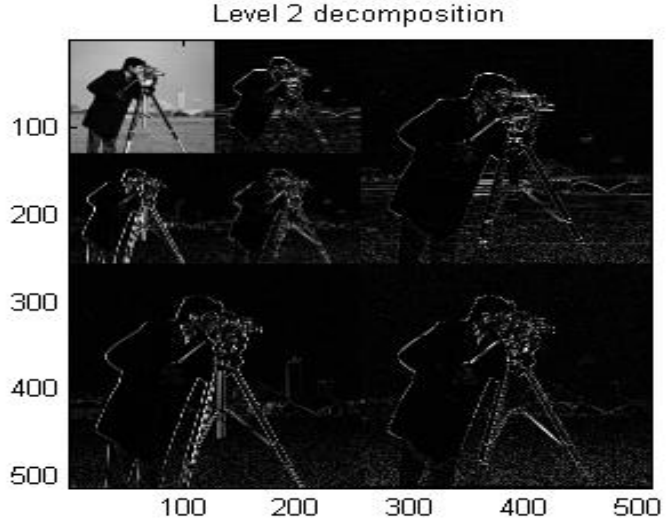

Fig:2 Image Decomposition(Level 2)

\section{PROPOSED APPROACH}

\subsection{Thresholding}

At different scales in the wavelet domain the transform coefficients have better decorrelation and localization property compared to the Discrete Fourier Transform (DFT) Coefficients. Thresholding is performed to remove the low energy coefficients as their contribution to the wavelet spectra is very low. There are two types of thresholding, Hard Thresholding and Soft thresholding. Hard thresholding sets any coefficient less than or equal to the threshold to zero as in the following:

$$
\text { if }(\operatorname{coef}[\mathrm{i}]<=\text { thresh }) \quad \operatorname{coef}[\mathrm{i}]=0.0 \text {; }
$$

In Soft thresholding, the threshold is subtracted from any coefficient that is greater than the threshold:

$$
\begin{aligned}
& \text { if }(\operatorname{coef}[\mathrm{i}]<=\text { thresh }) \operatorname{coef}[\mathrm{i}]=0.0 \text {; } \\
& \text { else } \operatorname{coef}[\mathrm{i}]=\text { coef }[\mathrm{i}]-\text { thresh; }
\end{aligned}
$$

Soft thresholding moves the time series toward zero.

For image denoising, soft threshold generally yields more visually pleasing results than hard threshold, and it is therefore the preferred choice.

\subsection{Methodology}

The methodology adopted here is the selection of the optimum threshold for wavelet transform compression scheme. The value of optimum threshold is calculated by comparing the visual quality of the compressed image. Here, a picture quality scale is used for measuring the visual quality of the image.

To obtain a picture quality scale (PQS) for the coding of achromatic images over the full range of image quality defined by the subjective mean opinion score (MOS) [14].

PQS takes into account the properties of visual perception for both global features and localized disturbances. Subjective assessment tests are widely used to evaluate the picture quality of coded images [15-17]. The PQS technique [18] uses distortion factors $\{\mathrm{Fi}\}$. Distortion factors are perceptually weighted measures of image impairments. The choice of the distortion factors is suggested by experience in observing artifacts due to coding and by knowledge of properties of the human visual system. Generally three distortion factors are chosen, Global distortion, distortion that produce texture patterns and local distortion around image contours. Regression methods are used to combine these factors into a single number representative of the quality of a given image. In each case, assumed that the nonlinear characteristic of the image display system has been compensated so that the image signal, $i(m, n)$ is equal to the luminance of the display at each pixel.

Table 1: Mean Opinion Score

\begin{tabular}{|ccc|}
\hline MOS & Quality & Impairment \\
5 & Excellent & Imperceptible \\
4 & Good & Perceptible but not annoying \\
3 & Fair & Slightly annoying \\
2 & Poor & Annoying \\
1 & Bad & Very annoying \\
\hline
\end{tabular}

Steps to determine the optimum threshold:

(1) Let default threshold $\lambda_{=} \lambda_{0}$, where $\lambda_{0}=0$.

(2) Choose, $\varepsilon=$ a small positive value, and $\mathrm{n}=0$

(3) $\mathrm{n}=\mathrm{n}+1 ; \lambda_{\mathrm{n}}=\lambda_{\mathrm{n}-1}+\varepsilon$

(4) Take Wavelet transform of the image using threshold $\lambda n$ and get coefficients $X(\lambda n)$.

(5) Reconstruct the image and Measure PQS and compare it with MOS Scale:

$$
\text { If acceptable, } \lambda \mathrm{opt}=\lambda_{\mathrm{n}} \text { and repeat step 3,4,5 }
$$

(6) If not acceptable then $\lambda$ opt $=\lambda_{\mathrm{n}}-\varepsilon$

(7)Output optimum threshold $\lambda$ opt.

(8)end

The above procedural steps are schematically represented in Fig(3).

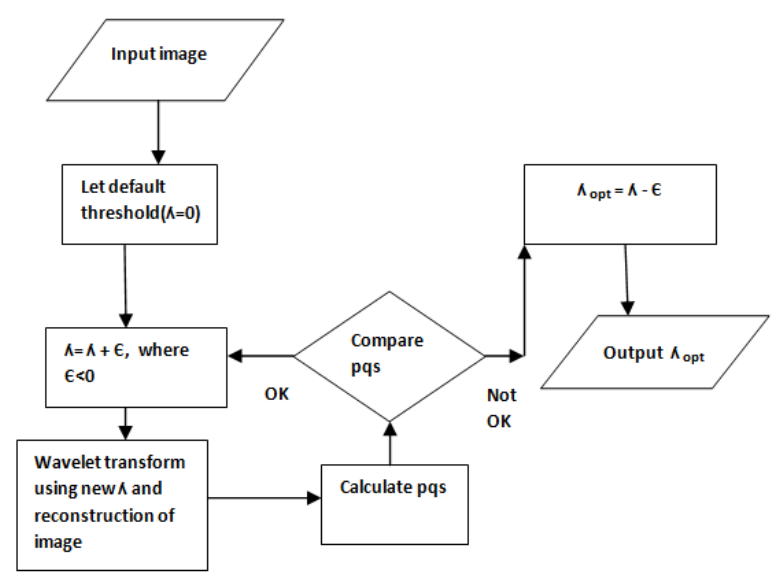

Fig:3 Block Schematic of proposed system

\section{RESULT AND DISCUSSIONS}

The proposed method has been tested with standard test images, cameraman, lena, jet, circuit and Peppers of size 256 X 256 and 8 bits/pixel. Figures 4(a),4(b),4(c) illustrate the test results. The results of optimum threshold obtained are listed in Table 2. When the results of the proposed approach are compared with the existing approach, the compression scores are found to be about two times that of the existing approach. Though PQS values are lower for the proposed approach, there is no significant reduction in the quality of the reconstructed image to the human eye. The PQS values attained are comparable with MOS as given in Table 1. 


\section{CONCLUSION}

In this paper, a method for computing optimum threshold for wavelet transform is proposed. The visual quality of the image is considered while computing the threshold. With the optimum threshold value, it is expected that maximum redundant information is removed from the input image resulting better compression and also high visual quality reconstructed image.

Table 2:Test Results

\begin{tabular}{|c|c|c|c|c|c|c|c|c|}
\hline $\begin{array}{l}\dot{Z} \\
\dot{\infty}\end{array}$ & 莕 & $\sum^{\frac{0}{0}}$ & 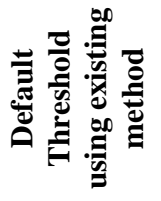 & 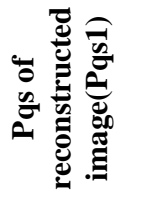 & 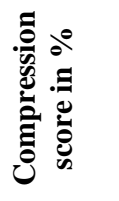 & 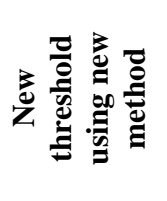 & 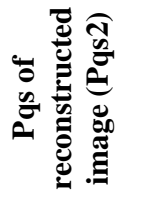 & 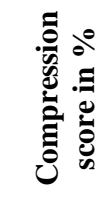 \\
\hline 1 & cameraman & haar & 1.5 & 5.0687 & 40.2252 & 7.4642 & 3.9428 & 72.7509 \\
\hline 2 & lena & haar & 2.5 & 4.7998 & 42.7002 & 9.093 & 3.2227 & 73.6710 \\
\hline 3 & circuit & $\mathrm{db} 2$ & 2 & 4.5346 & 51.6979 & 3.95 & 3.7921 & 67.5813 \\
\hline 4 & jet & $\mathrm{db} 2$ & 1.5 & 5.2450 & 40.1755 & 12.35 & 3.5524 & 88.0440 \\
\hline 5 & peppers & $\mathrm{db} 3$ & 2 & 5.0436 & 31.5457 & 7.4849 & 3.2534 & 75.8448 \\
\hline
\end{tabular}
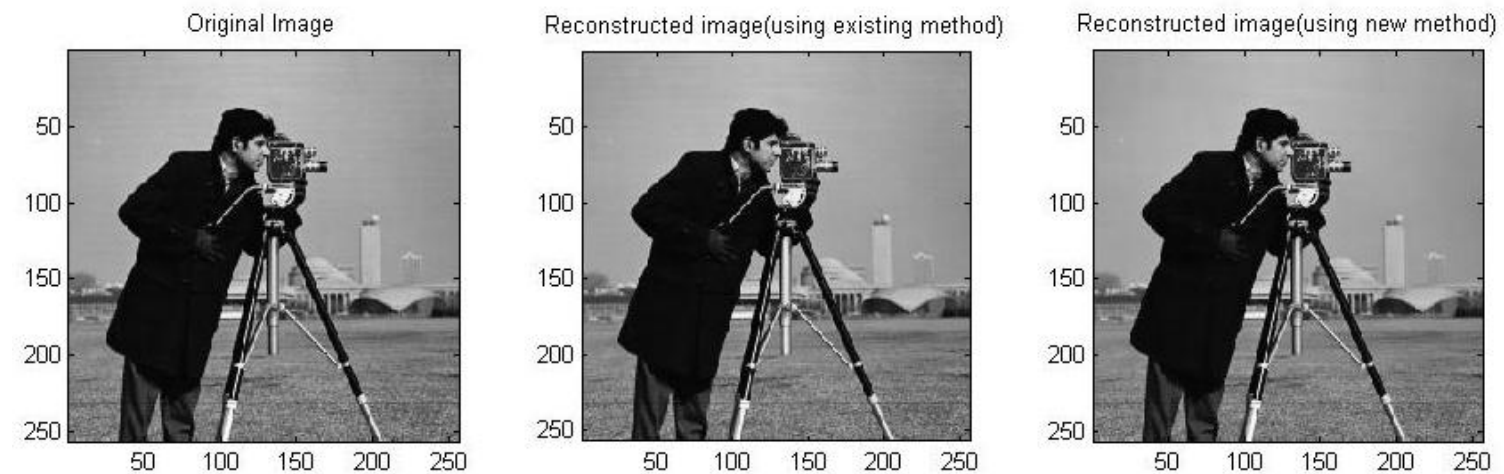

Fig: 4(a) Original and Reconstructed Images
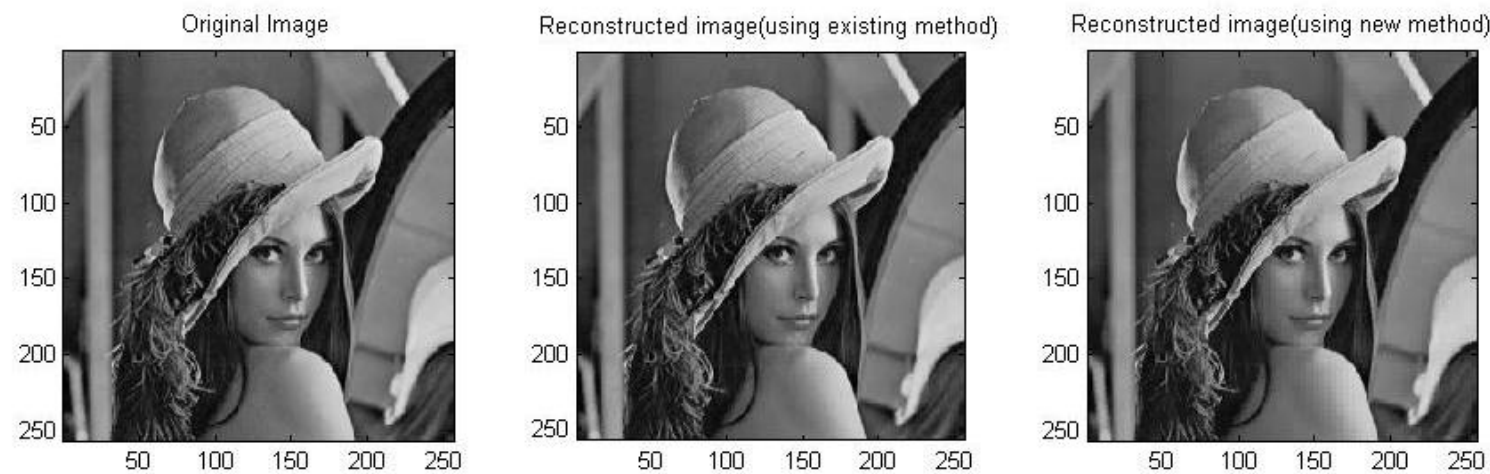

Fig: 4(b) Original and Reconstructed Images
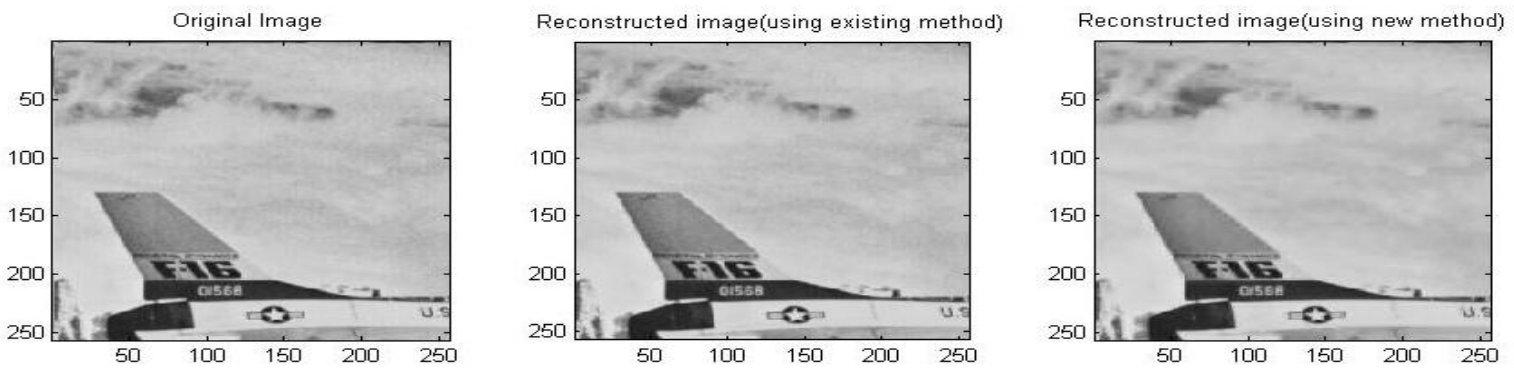

Fig: 4(c) Original and Reconstructed Images 


\section{REFERENCES}

[1] CUI Huimin, ZHAO Ruimei, HOU Yanli, Improved Threshold Denoising Method Based on Wavelet Transform, Elsevier, Physics Procedia 33 ( 2012 ) 1354 1359,2012

[2] G.Andria,F.Attivissimo,G.Cavone,N.Giaquinto,A.M.L.L anzolla Linear filtering of 2D wavelet coefficients for denoising ultrasound medical images Elsevier, SciVerse Science Direct, Measurement 45(2012)1792-1800,2012.

[3] D.L. Donoho, De-Noising by Soft Threshold, IEEE Trans. Info. Theory 43,pp. 933-936, 1993.

[4] D.L. Donoho and I.M. Johnstone, Adapting to unknown smoothness via wavelet shrinkage, Journal of American Statistical Assoc., Vol. 90, no. 432, pp. 1200-1224, Dec. 1995.

[5] S. Grace Chang, Bin Yu and M. Vattereli, Adaptive Wavelet Threshold for Image Denoising and Compression, IEEE Trans. for Multiple Noisy Image Copies, IEEE Trans. Image Processing, vol. 9, pp.16311635, Sept. 2000.

[6] S. Grace Chang, Bin Yu and M. Vattereli, Wavelet Threshold for Multiple Noisy Image Copies, IEEE Trans. Image Processing, vol. 9, pp.1631- 1635, Sept. 2000.

[7] S. Grace Chang, Bin Yu and M. Vattereli, Spatially Adaptive Wavelet Threshold with Context Modeling for Image Denoising,, IEEE Trans. Image Processing, vol. 9, pp. 1522-1530, Sept. 2000.

[8] Daubechies, Ten Lectures on Wavelets, Vol. 61 of Proc. CBMS-NSF Regional Conference Series in Applied Mathematics. Philadelphia, PA: SIAM, 1992.

[9] Fei Xiaoa, Yungang Zhanga "A Comparative Study on Thresholding Methods in Waveletbased Image
Denoising" Elsevier, Procedia Engineering 15 (2011) 3998 - 4003,2011.

[10] M. L. Hilton and R. T. Ogden Data Analytic Wavelet Threshold Selection in 2-D Signal Denoising" IEEE Transactions on signal processing, vol. 45, no. 2, february 1997.

[11] Weszka, J.S.; Nagel, R.N.; Rosenfeld, Threshold Selection Technique "Computers,IEEE Transactions on ,Volume C-23, Issue 12, Dec. 1974 Page(s):1322 - 1326

[12] Marc Antonini, Michel Barlaud, "Image Coding Using Wavelet Transform" IEEE Transactions on Image Processing, Vol. 1, No. 2, April 1992. pp. 205-220

[13] S.Mallat, "A theory for multiresolution signal decomposition: The wavelet representation", IEEE Transactions Pattern Analysis Machine Intelligence, Vol II pp. 674-693, 1989.

[14] Miyahara, M. ,Kotani, K. , Algazi, V., "Objective picture quality scale (PQS) for image coding" IEEE Transactions on Communications, Vol.46, Issue: 9 ,Sep 1998, pp. 1215-1226.

[15] N.W.Lewis and J.A.Alnatt "Subjective quality of television pictures with multiple impairments" Electron, Lett.,vol.,1,pp. 187-188,July

[16] Method for the subjective assessment of the quality of television pictures,CCIR Rec. 500-2,1982.

[17] "Advanced methods for the evaluation of television picture quality" in proc., Mosiac workshop, Eindhoven,The Netherlands, 1995.

[18] Makoto Miyahara, Kazunori Kotani, and V. Ralph Algazi.Objective picture quality Scale(PQS) for image coding IEEE Ttransactions on communications, vol. 46, no. 9 , september 1998 